\title{
Jet Tomography of Quark Gluon Plasma
}

\author{
Xin-Nian Wang \\ Nuclear Science Division, MS 70R0319, \\ Lawrence Berkeley National Laboratory, Berkeley, CA 94720
}

Received on 12 December, 2003

\begin{abstract}
Recent experimental measurements of high $p_{T}$ hadron spectra and jet correlation at RHIC are analyzed within a parton model which incoporates initial jet production and final propagation in heavy-ion collisions. The suppresion of single hadron spectra, back-to-back correlation, their centrality dependence and azimuthal anisotropy point to a dense matter with an initial parton density about 30 times of that in a cold heavy nucleus.
\end{abstract}

\section{Introduction}

In high-energy heavy-ion collisions, a dense medium of quarks and gluons is expected to be produced and possibly a quark-gluon plasma is formed. One important step in establishing evidence of QGP formation is to charaterize the properties of the dense medium produced, for example, the parton and energy density and color confinement, among many other charateristics. Conventionally, one can study the properties of a medium via scattering experiments with particle beams. In deeply inelastic scattering (DIS) experiments, for example, leptons scatter off the nucleon medium via photon exchange with quarks. The response function or the correlation function of the electromagnetic currents,

$$
W_{\mu \nu}(q)=\frac{1}{4 \pi} \int d^{4} x e^{i q \cdot x}\left\langle A\left|j_{\mu}^{e m}(0) j_{\nu}^{e m}(x)\right| A\right\rangle
$$

is a direct measurement of the quark distributions in a nucleon or nucleus. Such experiments have provided unprecedented information about partonic structure of nucleons and nucleus and confirmed the prediction of QCD evolution[1].

For dynamic systems such as that produced in heavy-ion collisions, one can no longer use the technique of scattering with a beam of particles because of the transient nature of the matter. The lifetime of the system is very short, on the order of a few $\mathrm{fm} / c$. The initial spatial size is only the size of the heaviest nuclei, about $6 \mathrm{fm}$ in diameter in the transverse dimension. The system expands very rapidly both in the longitudinal and transverse direction. These characteristics make it impossible to use external probes to study the properties of the produced dense matter in high-energy heavyion collisions. Fortunately, one can prove that the thermal average of the above correlation function gives the photon emission rate from the evolving system. The emission rate depends mainly on the local temperature or the parton density while the total yield also depends the whole evolution history of the system. Therefore, a strongly interacting system can reveal its properties and dynamics through photon and dilepton emission. One can further study the resonance properties of the emitted virtual photons and their medium modification. The screening of strong interaction in a color deconfined medium should lead to dissociation of the binding states and thus the quarkonia suppression [2]. Such color screening is a result of strong interaction between quarks and gluons at high density and temperature. The same interaction will also cause attenuation of fast and energetic partons propagating through the medium. Such an effect is the underlying physics of the jet quenching [3] phenomenon and jet tomography technique for studying properties of dense matter in high-energy heavy-ion collisions.

Jet quenching as a probe of the dense matter in heavy-ion collisions, takes advantage of the hard processes of jet production in high-energy heavy-ion collisions. Similar to the technology of computed tomography (CT), study of these energetic particles, their initial production and interaction with the dense medium, can yield critical information about the properties of the matter that is otherwise difficult to access through soft hadrons from the hadronization of the bulk medium. Though relatively rare with small cross sections, the jet production rate can be calculated perturbative in QCD and agrees well with experimental measurements in highenergy $p p(\bar{p})$ collisions. A critical component of the jet tomography is then to understand the jet attenuation through dense matter as it propagates through the medium.

\section{Modified Fragmentation Function}

A direct manifest of jet quenching is the modification of the fragmentation function of the produced parton, $D_{a \rightarrow h}\left(z, \mu^{2}\right)$ which can be measured directly. This modification can be directly translated into the energy loss of the leading parton.

To demonstrate medium modified fragmentation function and parton energy loss, one can study deeply inelastic scattering (DIS) $e A[4,5,6]$. Here, we consider the semiinclusive processes, $e\left(L_{1}\right)+A(p) \longrightarrow e\left(L_{2}\right)+h\left(\ell_{h}\right)+X$, where $L_{1}$ and $L_{2}$ are the four-momenta of the incoming and the outgoing leptons, and $\ell_{h}$ is the observed hadron momentum. The differential cross section for the semi-inclusive 
process can be expressed as

$$
E_{L_{2}} E_{\ell_{h}} \frac{d \sigma_{\mathrm{DIS}}^{h}}{d^{3} L_{2} d^{3} \ell_{h}}=\frac{\alpha_{\mathrm{EM}}^{2}}{2 \pi s} \frac{1}{Q^{4}} L_{\mu \nu} E_{\ell_{h}} \frac{d W^{\mu \nu}}{d^{3} \ell_{h}},
$$

where $p=\left[p^{+}, 0, \mathbf{0}_{\perp}\right]$ is the momentum per nucleon in the nucleus, $q=L_{2}-L_{1}=\left[-Q^{2} / 2 q^{-}, q^{-}, \mathbf{0}_{\perp}\right]$ the momentum transfer, $s=\left(p+L_{1}\right)^{2}$ and $\alpha_{\mathrm{EM}}$ is the electromagnetic (EM) coupling constant. $L_{\mu \nu}$ is the leptonic tensor while $W_{\mu \nu}$ is the semi-inclusive hadronic tensor.

In the parton model with the collinear factorization approximation, the leading-twist contribution to the semiinclusive cross section can be factorized into a product of parton distributions, parton fragmentation functions and the hard partonic cross section. Including all leading log radiative corrections, the lowest order contribution from a single hard $\gamma^{*}+q$ scattering can be written as

$$
\begin{aligned}
\frac{d W_{\mu \nu}^{S}}{d z_{h}} & =\sum_{q} e_{q}^{2} \int d x f_{q}^{A}\left(x, \mu_{I}^{2}\right) H_{\mu \nu}^{(0)}(x, p, q) \\
& \times D_{q \rightarrow h}\left(z_{h}, \mu^{2}\right)
\end{aligned}
$$

where $H_{\mu \nu}^{(0)}(x, p, q)$ is the hard part of the process in leading order, the momentum fraction carried by the hadron is defined as $z_{h}=\ell_{h}^{-} / q^{-}$and $x_{B}=Q^{2} / 2 p^{+} q^{-}$is the Bjorken variable. $\mu_{I}^{2}$ and $\mu^{2}$ are the factorization scales for the initial quark distributions $f_{q}^{A}\left(x, \mu_{I}^{2}\right)$ in a nucleus and the fragmentation functions $D_{q \rightarrow h}\left(z_{h}, \mu^{2}\right)$, respectively.

In a nuclear medium, the propagating quark in DIS will experience additional scatterings with other partons from the nucleus. The rescatterings may induce additional gluon radiation and cause the leading quark to lose energy. Such induced gluon radiations will effectively give rise to additional terms in the evolution equation leading to the modification of the fragmentation functions in a medium. These are the so-called higher-twist corrections since they involve highertwist parton matrix elements and are power-suppressed. We will consider those contributions that involve two-parton correlations from two different nucleons inside the nucleus.

One can apply the generalized factorization to these multiple scattering processes[7]. In this approximation, the double scattering contribution to radiative correction can be calculated and the effective modified fragmentation function is

$$
\begin{aligned}
\widetilde{D}_{q \rightarrow h}\left(z_{h}, \mu^{2}\right) \equiv & D_{q \rightarrow h}\left(z_{h}, \mu^{2}\right)+\int_{0}^{\mu^{2}} \frac{d \ell_{T}^{2}}{\ell_{T}^{2}} \frac{\alpha_{s}}{2 \pi} \int_{z_{h}}^{1} \frac{d z}{z} \\
& {\left[\Delta \gamma_{q \rightarrow q g}\left(z, x, x_{L}, \ell_{T}^{2}\right) D_{q \rightarrow h}\left(z_{h} / z\right)\right.} \\
+ & \left.\Delta \gamma_{q \rightarrow g q}\left(z, x, x_{L}, \ell_{T}^{2}\right) D_{g \rightarrow h}\left(z_{h} / z\right)\right]
\end{aligned}
$$

where $D_{q \rightarrow h}\left(z_{h}, \mu^{2}\right)$ and $D_{g \rightarrow h}\left(z_{h}, \mu^{2}\right)$ are the leading-twist fragmentation functions. The modified splitting functions are given as

$$
\begin{aligned}
& \Delta \gamma_{q \rightarrow q g}\left(z, x, x_{L}, \ell_{T}^{2}\right)=\left[\frac{1+z^{2}}{(1-z)_{+}} T_{q g}^{A}\left(x, x_{L}\right)\right. \\
&\left.+\delta(1-z) \Delta T_{q g}^{A}\left(x, \ell_{T}^{2}\right)\right] \frac{2 \pi \alpha_{s} C_{A}}{\ell_{T}^{2} N_{c} f_{q}^{A}\left(x, \mu_{I}^{2}\right)} \\
& \Delta \gamma_{q \rightarrow g q}\left(z, x, x_{L}, \ell_{T}^{2}\right)+\Delta \gamma_{q \rightarrow q g}\left(1-z, x, x_{L}, \ell_{T}^{2}\right) .
\end{aligned}
$$

Here, the fractional momentum is defined as $x_{L}=\ell_{T}^{2} / 2 p^{+} q^{-} z(1-z)$ and $x=x_{B}=Q^{2} / 2 p^{+} q^{-}$is the Bjorken variable. The twist-four parton matrix elements of the nucleus,

$$
\begin{aligned}
T_{q g}^{A}\left(x, x_{L}\right) & =\int \frac{d y^{-}}{2 \pi} d y_{1}^{-} d y_{2}^{-} e^{i\left(x+x_{L}\right) p^{+} y^{-}}\left(1-e^{-i x_{L} p^{+} y_{2}^{-}}\right) \\
\times & \left(1-e^{-i x_{L} p^{+}\left(y^{-}-y_{1}^{-}\right)}\right) \theta\left(-y_{2}^{-}\right) \theta\left(y^{-}-y_{1}^{-}\right) \\
\times & \frac{1}{2}\left\langle A\left|\bar{\psi}_{q}(0) \gamma^{+} F_{\sigma}^{+}\left(y_{2}^{-}\right) F^{+\sigma}\left(y_{1}^{-}\right) \psi_{q}\left(y^{-}\right)\right| A\right\rangle
\end{aligned}
$$

has a dipole-like structure which is a result of LandauPomerachuck-Migdal (LPM) interference in gluon bremsstrahlung. Here the intrinsic transverse momentum is neglected. In the limit of collinear radiation $\left(x_{L} \rightarrow 0\right)$ or when the formation time of the gluon radiation, $\tau_{f} \equiv 1 / x_{L} p^{+}$, is much larger than the nuclear size, the destructive interference leads to the LPM interference effect.

Using the factorization approximation $[4,5,7,8]$, we can relate the twist-four parton matrix elements of the nucleus to the twist-two parton distributions of nucleons and the nu- 
cleus,

$$
T_{q g}^{A}\left(x, x_{L}\right) \approx \frac{\widetilde{C}}{x_{A}}\left(1-e^{-x_{L}^{2} / x_{A}^{2}}\right) f_{q}^{A}(x),
$$

where $\widetilde{C} \equiv 2 C x_{T} f_{g}^{N}\left(x_{T}\right)$ is considered a constant. One can identify $1 / x_{L} p^{+}=2 q^{-} z(1-z) / \ell_{T}^{2}$ as the formation time of the emitted gluons. When it becomes comparable or larger than the nuclear size, the above matrix element vanishes, demonstrating a typical LPM interference effect.

Since the LPM interference suppresses gluon radiation whose formation time $\left(\tau_{f} \sim Q^{2} / \ell_{T}^{2} p^{+}\right)$is larger than the nuclear size $M R_{A} / p^{+}$in our chosen frame, $\ell_{T}^{2}$ should then have a minimum value of $\ell_{T}^{2} \sim Q^{2} / M R_{A} \sim Q^{2} / A^{1 / 3}$. Here $M$ is the nucleon mass. Therefore, the leading highertwist contribution is proportional to $\alpha_{s} R_{A} / \ell_{T}^{2} \sim \alpha_{s} R_{A}^{2} / Q^{2}$ due to double scattering and depends quadratically on the nuclear size $R_{A}$.

With the assumption of the factorized form of the twist4 nuclear parton matrices, there is only one free parameter $\widetilde{C}\left(Q^{2}\right)$ which represents quark-gluon correlation strength inside nuclei. Once it is fixed, one can predict the $z$, energy and nuclear dependence of the medium modification of the fragmentation function. Shown in Fig. 1 are the calculated nuclear modification factor of the fragmentation functions for ${ }^{14} \mathrm{~N}$ and ${ }^{84} \mathrm{Kr}$ targets as compared to the recent HERMES data[9]. The predicted shape of the $z$-dependence agrees well with the experimental data. A remarkable feature of the prediction is the quadratic $A^{2 / 3}$ nuclear size dependence, which is verified for the first time by an experiment. By fitting the overall suppression for one nuclear target, we obtain the only parameter in our calculation, $\widetilde{C}\left(Q^{2}\right)=0.0060 \mathrm{GeV}^{2}$ with $\alpha_{\mathrm{s}}\left(Q^{2}\right)=0.33$ at $Q^{2} \approx 3$ $\mathrm{GeV}^{2}$. The predicted $\nu$-dependence also agrees with the experimental data [22].

We can quantify the modification of the fragmentation by the quark energy loss which is defined as the momentum fraction carried by the radiated gluon,

$$
\begin{aligned}
\left\langle\Delta z_{g}\right\rangle & =\int_{0}^{\mu^{2}} \frac{d \ell_{T}^{2}}{\ell_{T}^{2}} \int_{0}^{1} d z \frac{\alpha_{s}}{2 \pi} z \Delta \gamma_{q \rightarrow g q}\left(z, x_{B}, x_{L}, \ell_{T}^{2}\right) \\
& =\widetilde{C} \frac{C_{A} \alpha_{s}^{2}}{N_{c}} \frac{x_{B}}{x_{A} Q^{2}} \int_{0}^{1} d z \frac{1+(1-z)^{2}}{z(1-z)} \\
& \times \int_{0}^{x_{\mu}} \frac{d x_{L}}{x_{L}^{2}}\left(1-e^{-x_{L}^{2} / x_{A}^{2}}\right)
\end{aligned}
$$

where $x_{\mu}=\mu^{2} / 2 p^{+} q^{-} z(1-z)=x_{B} / z(1-z)$ if we choose the factorization scale as $\mu^{2}=Q^{2}$. When $x_{A} \ll x_{B} \ll 1$ we can estimate the leading quark energy loss roughly as

$$
\left\langle\Delta z_{g}\right\rangle\left(x_{B}, \mu^{2}\right) \approx \widetilde{C} \frac{C_{A} \alpha_{s}^{2}}{N_{c}} \frac{x_{B}}{Q^{2} x_{A}^{2}} 6 \sqrt{\pi} \ln \frac{1}{2 x_{B}} .
$$

Since $x_{A}=1 / M R_{A}$, the energy loss $\left\langle\Delta z_{g}\right\rangle$ thus depends quadratically on the nuclear size.

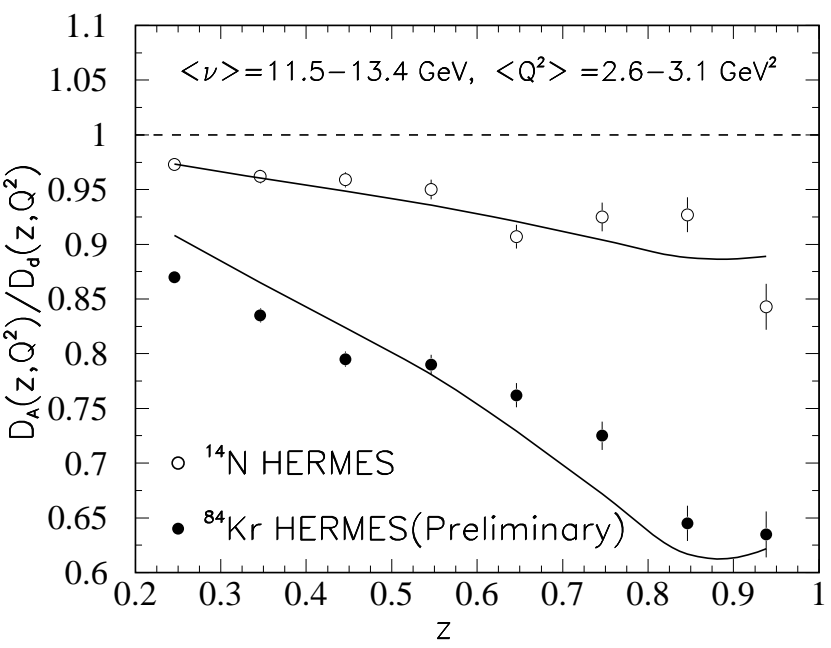

Figure 1. Predicted nuclear modification of jet fragmentation function is compared to the HERMES data [9] on ratios of hadron distributions between $A$ and $d$ targets in DIS.

In the rest frame of the nucleus, $p^{+}=m_{N}, q^{-}=$ $\nu$, and $x_{B} \equiv Q^{2} / 2 p^{+} q^{-}=Q^{2} / 2 m_{N} \nu$. One can get the averaged total energy loss as $\Delta E=\nu\left\langle\Delta z_{g}\right\rangle \approx$ $\widetilde{C}\left(Q^{2}\right) \alpha_{\mathrm{s}}^{2}\left(Q^{2}\right) m_{N} R_{A}^{2}\left(C_{A} / N_{c}\right) 3 \ln \left(1 / 2 x_{B}\right)$. With the determined value of $\widetilde{C},\left\langle x_{B}\right\rangle \approx 0.124$ in the HERMES experiment[9] and the average distance $\left\langle L_{A}\right\rangle=R_{A} \sqrt{2 / \pi}$ for the assumed Gaussian nuclear distribution, one gets the quark energy loss $d E / d L \approx 0.5 \mathrm{GeV} / \mathrm{fm}$ inside a $A u$ nucleus.

\section{Jet Quenching in Hot Medium at RHIC}

To extend our study of modified fragmentation functions to jets in heavy-ion collisions, we can assume $\left\langle k_{T}^{2}\right\rangle \approx \mu^{2}$ (the Debye screening mass) and a gluon density profile $\rho(y)=\left(\tau_{0} / \tau\right) \theta\left(R_{A}-y\right) \rho_{0}$ for a 1-dimensional expanding system. Since the initial jet production rate is independent of the final gluon density which can be related to the partongluon scattering cross section[10] $\left[\alpha_{s} x_{T} G\left(x_{T}\right) \sim \mu^{2} \sigma_{g}\right]$, one has then

$$
\frac{\alpha_{s} T_{q g}^{A}\left(x_{B}, x_{L}\right)}{f_{q}^{A}\left(x_{B}\right)} \sim \mu^{2} \int d y \sigma_{g} \rho(y)\left[1-\cos \left(y / \tau_{f}\right)\right],
$$

where $\tau_{f}=2 E z(1-z) / \ell_{T}^{2}$ is the gluon formation time. One can recover the form of energy loss in a thin plasma obtained in the opacity expansion approach[11], 


$$
\begin{aligned}
\left\langle\Delta z_{g}\right\rangle & =\frac{C_{A} \alpha_{s}}{\pi} \int_{0}^{1} d z \int_{0}^{\frac{Q^{2}}{\mu^{2}}} d u \frac{1+(1-z)^{2}}{u(1+u)} \int_{\tau_{0}}^{R_{A}} d \tau \sigma_{g} \rho(\tau) \\
& \times\left[1-\cos \left(\frac{\left(\tau-\tau_{0}\right) u \mu^{2}}{2 E z(1-z)}\right)\right]
\end{aligned}
$$

Keeping only the dominant contribution and assuming $\sigma_{g} \approx C_{a} 2 \pi \alpha_{s}^{2} / \mu^{2}\left(C_{a}=1\right.$ for $q g$ and $9 / 4$ for $g g$ scattering), one obtains the averaged energy loss,

$$
\left\langle\frac{d E}{d L}\right\rangle \approx \frac{\pi C_{a} C_{A} \alpha_{s}^{3}}{R_{A}} \int_{\tau_{0}}^{R_{A}} d \tau \rho(\tau)\left(\tau-\tau_{0}\right) \ln \frac{2 E}{\tau \mu^{2}} .
$$

Neglecting the logarithmic dependence on $\tau$, the averaged energy loss in a 1-dimensional expanding system can be expressed as $\left\langle\frac{d E}{d L}\right\rangle_{1 d} \approx\left(d E_{0} / d L\right)\left(2 \tau_{0} / R_{A}\right)$, where $d E_{0} / d L \propto \rho_{0} R_{A}$ is the energy loss in a static medium with the same gluon density $\rho_{0}$ as in a 1-d expanding system at time $\tau_{0}$. Because of the expansion, the averaged energy loss $\langle d E / d L\rangle_{1 d}$ is suppressed as compared to the static case and does not depend linearly on the system size.

In order to calculate the effects of parton energy loss on the attenuation pattern of high $p_{T}$ partons in nuclear collisions, we use a simpler effective modified fragmentation function[12, 13],

$$
\begin{gathered}
D_{h / c}^{\prime}\left(z_{c}, Q^{2}, \Delta E_{c}\right)=e^{-\left\langle\frac{\Delta L}{\lambda}\right\rangle} D_{h / c}^{0}\left(z_{c}, Q^{2}\right)+\left(1-e^{-\left\langle\frac{\Delta L}{\lambda}\right\rangle}\right) \\
\times\left[\frac{z_{c}^{\prime}}{z_{c}} D_{h / c}^{0}\left(z_{c}^{\prime}, Q^{2}\right)+\left\langle\frac{\Delta L}{\lambda}\right\rangle \frac{z_{g}^{\prime}}{z_{c}} D_{h / g}^{0}\left(z_{g}^{\prime}, Q^{2}\right)\right]
\end{gathered}
$$

where $z_{c}^{\prime}, z_{g}^{\prime}$ are the rescaled momentum fractions. This effective model is found to reproduce the pQCD result from Eq.(4) very well, but only when $\Delta z=\Delta E_{c} / E$ is set to be $\Delta z \approx 0.6\left\langle z_{g}\right\rangle$. Therefore the actual averaged parton energy loss should be $\Delta E / E=1.6 \Delta z$ with $\Delta z$ extracted from the effective model. The factor 1.6 is mainly caused by the unitarity correction effect in the pQCD calculation.

Since gluons are bosons, there should also be stimulated gluon emission and absorption by the propagating parton because of the presence of thermal gluons in the hot medium. Such detailed balance is crucial for parton thermalization and should also be important for calculating the energy loss of an energetic parton in a hot medium[14]. Taking into account such detailed balance in gluon emission, one can then get the asymptotic behavior of the effective energy loss in the opacity expansion framework [14],

$$
\begin{aligned}
\frac{\Delta E}{E} \approx & \frac{\alpha_{s} C_{F} \mu^{2} L^{2}}{4 \lambda_{g} E}\left[\ln \frac{2 E}{\mu^{2} L}-0.048\right] \\
& -\frac{\pi \alpha_{s} C_{F}}{3} \frac{L T^{2}}{\lambda_{g} E^{2}}\left[\ln \frac{\mu^{2} L}{T}-1+\gamma_{\mathrm{E}}-\frac{6 \zeta^{\prime}(2)}{\pi^{2}}\right](15)
\end{aligned}
$$

where the first term is from the induced bremsstralung and the second term is due to gluon absorption in detailed balance which effectively reduce the total parton energy loss in the medium. Numerical calculations show that the effect of the gluon absorption is small and can be neglected for par- tons with very high energy. However, the thermal absorption reduces the effective parton energy loss by about 30-10\% for intermediate values of parton energy. This will increase the energy dependence of the effective parton energy loss in the intermediate energy region. One can parameterize such energy dependence as,

$$
\left\langle\frac{d E}{d L}\right\rangle_{1 d}=\epsilon_{0}(E / \mu-1.6)^{1.2} /(7.5+E / \mu),
$$

The threshold is the consequence of gluon absorption that competes with radiation that effectively shuts off the energy loss. The parameter $\mu$ is set to be $1 \mathrm{GeV}$ in the calculation.

To calculate the modified high $p_{T}$ spectra in $A+A$ collisions, we use a LO pQCD model $[15,16]$,

$$
\begin{aligned}
\frac{d \sigma_{A A}^{h}}{d y d^{2} p_{T}} & =K \sum_{a b c d} \int d^{2} b d^{2} r d x_{a} d x_{b} d^{2} k_{a T} d^{2} k_{b T} \\
& \times t_{A}(r) t_{A}(|\mathbf{b}-\mathbf{r}|) g_{A}\left(k_{a T}, r\right) g_{A}\left(k_{b T},|\mathbf{b}-\mathbf{r}|\right) \\
& \times f_{a / A}\left(x_{a}, Q^{2}, r\right) f_{b / A}\left(x_{b}, Q^{2},|\mathbf{b}-\mathbf{r}|\right) \\
& \times \frac{D_{h / c}^{\prime}\left(z_{c}, Q^{2}, \Delta E_{c}\right)}{\pi z_{c}} \frac{d \sigma}{d \hat{t}}(a b \rightarrow c d)
\end{aligned}
$$

with medium modified fragmentation funcitons $D_{h / c}^{\prime}$ given by Eq. (14) and the fragmentation functions in free space $D_{h / c}^{0}\left(z_{c}, Q^{2}\right)$ are given by the BBK parameterization [17]. Here, $z_{c}=p_{T} / p_{T c}, y=y_{c}, \sigma(a b \rightarrow c d)$ are elementary parton scattering cross sections and $t_{A}(b)$ is the nuclear 
thickness function normalized to $\int d^{2} b t_{A}(b)=A$. We will use a hard-sphere model of nuclear distribution in this paper. The $K \approx 1.5-2$ factor is used to account for higher order pQCD corrections.

The parton distributions per nucleon $f_{a / A}\left(x_{a}, Q^{2}, r\right)$ inside the nucleus are assumed to be factorizable into the parton distributions in a free nucleon given by the MRSD- ${ }^{\prime}$ parameterization [18] and the impact-parameter dependent nuclear modification factor which will given by the new HIJING parameterization [19]. The initial transverse momentum distribution $g_{A}\left(k_{T}, Q^{2}, b\right)$ is assumed to have a Gaussian form with a width that includes both an intrinsic part in a nucleon and nuclear broadening. This model has been fitted to the nuclear modification of the $p_{T}$ spectra in $p+A$ collisions at up to the Fermilab energy $\sqrt{s}=40 \mathrm{GeV}$. Shown in Fig. 2 are the first prediction made in 1998 [15] of the Cronin effect at RHIC for $p+A u$ collisions at $\sqrt{s}=200 \mathrm{GeV}$ as compared to the recent RHIC data. As one can see, the initial multiple scattering in nuclei can give some moderate Cronin enhancement of the high $p_{T}$ spectra. Therefore, any suppression of the high $p_{T}$ spectra in $A u+A u$ collisions has to be caused by jet quenching.

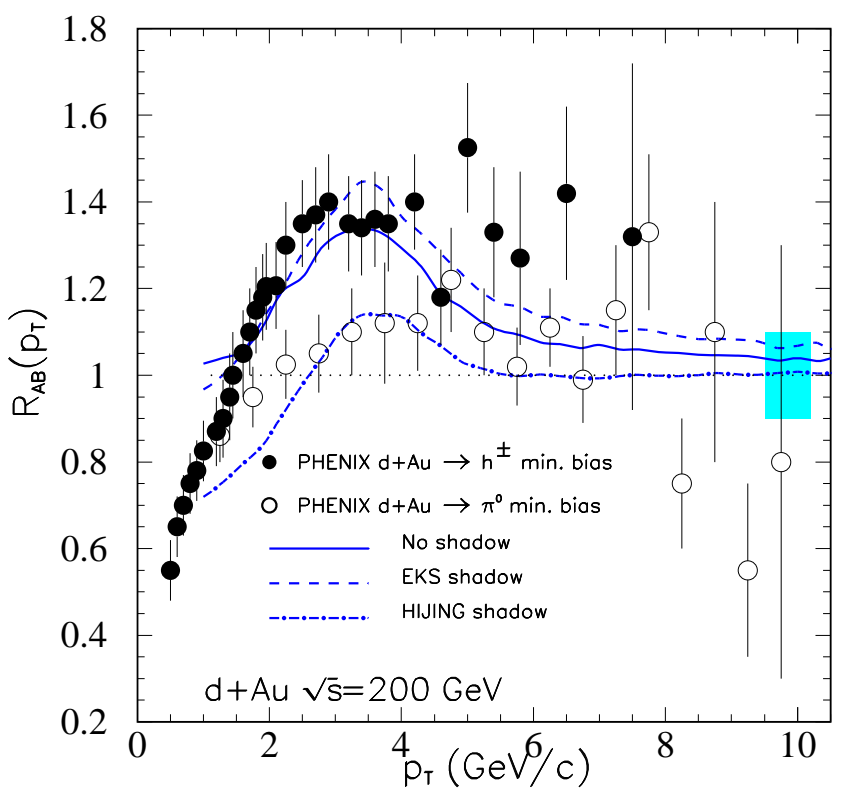

Figure 2. The first predictions [15] of the Cronin effect in $p+A u$ collisions at $\sqrt{s}=200 \mathrm{GeV}$ are compared to the recent RHIC data from PHENIX [20] and STAR [21].

We assume a 1-dimensional expanding medium with a gluon density $\rho_{g}(\tau, r)$ that is proportional to the transverse profile of participant nucleons. According to Eq. 13, we will calculate impact-parameter dependence of the energy loss as

$$
\Delta E(b, r, \phi) \approx\left\langle\frac{d E}{d L}\right\rangle_{1 d} \int_{\tau_{0}}^{\Delta L} d \tau \frac{\tau-\tau_{0}}{\tau_{0} \rho_{0}} \rho_{g}(\tau, b, \vec{r}+\vec{n} \tau),
$$

where $\Delta L(b, \vec{r}, \phi)$ is the distance a jet, produced at $\vec{r}$, has to travel along $\vec{n}$ at an azimuthal angle $\phi$ relative to the reaction plane in a collision with impact-parameter $b$. Here, $\rho_{0}$ is the averaged initial gluon density at $\tau_{0}$ in a central collision and $\langle d E / d L\rangle_{1 d}$ is the average parton energy loss over a distance $R_{A}$ in a 1-d expanding medium with an initial uniform gluon density $\rho_{0}$. The corresponding energy loss in a static medium with a uniform gluon density $\rho_{0}$ over a distance $R_{A}$ is [22] $d E_{0} / d L=\left(R_{A} / 2 \tau_{0}\right)\langle d E / d L\rangle_{1 d}$. We will use the parameterization in Eq. (16) for the effective energy dependence of the parton quark energy loss.

Shown in Fig. 3 are the calculated nuclear modification factors $R_{A B}\left(p_{T}\right)=d \sigma_{A B}^{h} /\left\langle N_{\text {binary }}\right\rangle d \sigma_{p p}^{h}$ for hadron spectra $(|y|<0.5)$ in $A u+A u$ collisions at $\sqrt{s}=200$ $\mathrm{GeV}$, as compared to experimental data [24, 23]. Here, $\left\langle N_{\text {binary }}\right\rangle=\int d^{2} b d^{2} r t_{A}(r) t_{A}(|\vec{b}-\vec{r}|)$. To fit the observed $\pi^{0}$ suppression (solid lines) in the most central collisions, we have used $\mu=1.5 \mathrm{GeV}, \epsilon_{0}=1.07 \mathrm{GeV} / \mathrm{fm}$ and $\lambda_{0}=1 /\left(\sigma \rho_{0}\right)=0.3 \mathrm{fm}$. The hatched area (also in other figures in this paper) indicates a variation of $\epsilon_{0}= \pm 0.3$ $\mathrm{GeV} / \mathrm{fm}$. The hatched boxes around $R_{A B}=1$ represent experimental errors in overall normalization. Nuclear $k_{T}$ broadening and parton shadowing together give a slight enhancement of hadron spectra at intermediate $p_{T}=2-4 \mathrm{GeV} / c$ without parton energy loss.

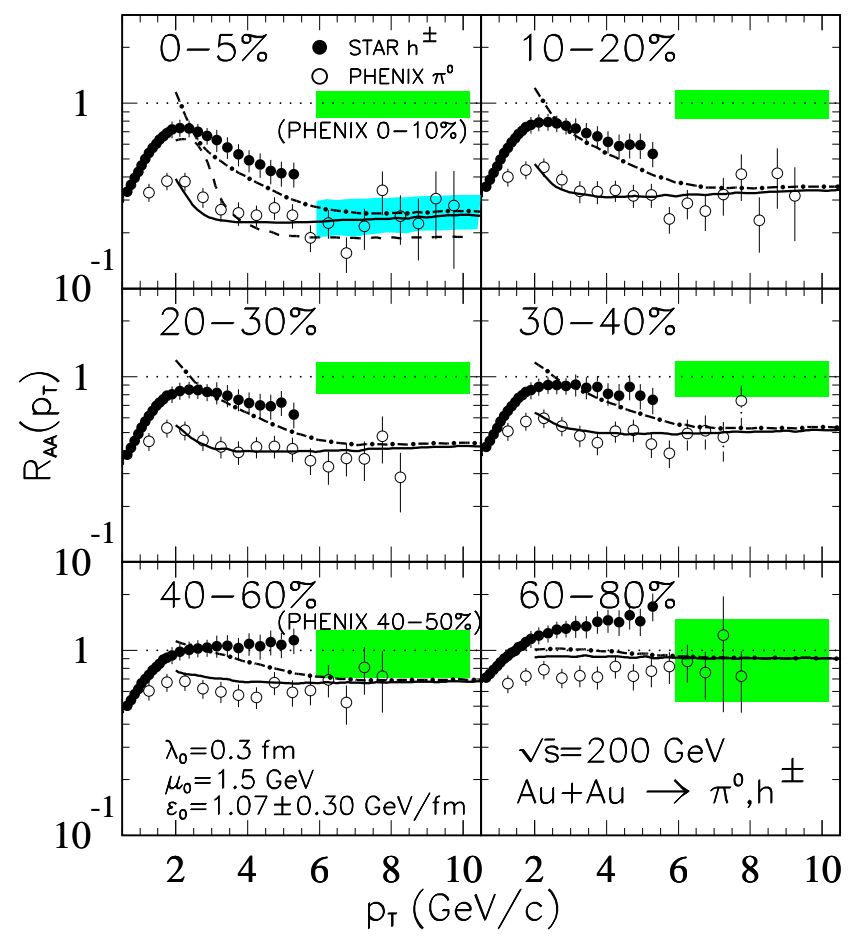

Figure 3. Hadron suppression factors in $A u+A u$ collisions as compared to data from STAR[24] and PHENIX [23]. See text for a detailed explanation.

The flat $p_{T}$ dependence of the $\pi^{0}$ suppression is a consequence of the strong energy dependence of the parton energy loss. The slight rise of $R_{A B}$ at $p_{T}<4 \mathrm{GeV} / c$ in the calculation is due to the detailed balance effect in the effective parton energy loss. In this region, one expects the fragmentation picture to gradually lose its validity and is taken over by other non-perturbative effects, especially for kaons 
and baryons. As a consequence, the $(K+p) / \pi$ ratio in central $A u+A u$ collisions is significantly larger than in peripheral $A u+A u$ or $p+p$ collisions. To take into account this effect, we add a nuclear dependent (proportional to $\left\langle N_{\text {binary }}\right\rangle$ ) soft component to kaon and baryon fragmentation functions so that $(K+p) / \pi \approx 2$ at $p_{T} \sim 3 \mathrm{GeV} / c$ in the most central $A u+A u$ collisions and approaches its $p+p$ value at $p_{T}>5 \mathrm{GeV} / c$. The resultant suppression for total charged hadrons (dot-dashed) and the centrality dependence agree well with the STAR data. One can direc- tly relate $h^{ \pm}$and $\pi^{0}$ suppression via the $(K+p) / \pi$ ratio: $R_{A A}^{h^{ \pm}}=R_{A A}^{\pi^{0}}[1+(K+p) / \pi]_{A A} /[1+(K+p) / \pi]_{p p}$. It is clear from the data that $(K+p) / \pi$ becomes the same for $A u+A u$ and $p+p$ collisions at $p_{T}>5 \mathrm{GeV} / c$. To demonstrate the sensitivity to the parameterized parton energy loss in the intermediate $p_{T}$ region, we also show $R_{A A}^{h^{ \pm}}$in 0 $5 \%$ centrality (dashed line) for $\mu=2.0 \mathrm{GeV}$ and $\epsilon_{0}=2.04$ $\mathrm{GeV} / \mathrm{fm}$ without the soft component.

In the same LO pQCD parton model, one can also calculate di-hadron spectra,

$$
\begin{aligned}
& E_{1} E_{2} \frac{d \sigma_{A A}^{h_{1} h_{2}}}{d^{3} p_{1} d^{3} p_{2}}=\frac{K}{2} \sum_{a b c d} \int d^{2} b d^{2} r d x_{a} d x_{b} d^{2} k_{a T} d^{2} k_{b T} \\
\times \quad & t_{A}(r) t_{A}(|\mathbf{b}-\mathbf{r}|) g_{A}\left(k_{a T}, r\right) g_{A}\left(k_{b T},|\mathbf{b}-\mathbf{r}|\right) \\
\times \quad & f_{a / A}\left(x_{a}, Q^{2}, r\right) f_{b / A}\left(x_{b}, Q^{2},|\mathbf{b}-\mathbf{r}|\right) \\
\times \quad & D_{h / c}\left(z_{c}, Q^{2}, \Delta E_{c}\right) D_{h / d}\left(z_{d}, Q^{2}, \Delta E_{d}\right) \frac{\hat{s}}{2 \pi z_{c}^{2} z_{d}^{2}} \\
\times \quad & \frac{d \sigma}{d \hat{t}}(a b \rightarrow c d) \delta^{4}\left(p_{a}+p_{b}-p_{c}-p_{d}\right),
\end{aligned}
$$

for two back-to-back hadrons from independent fragmentation of the back-to-back jets. Let us assume hadron $h_{1}$ is a triggered hadron with $p_{T 1}=p_{T}^{\text {trig }}$. One can define a hadrontriggered fragmentation function (FF) as the back-to-back correlation with respect to the triggered hadron:

$$
D^{h_{1} h_{2}}\left(z_{T}, \phi, p_{T}^{\mathrm{trig}}\right)=p_{T}^{\mathrm{trig}} \frac{d \sigma_{A A}^{h_{1} h_{2}} / d^{2} p_{T}^{\text {trig }} d p_{T} d \phi}{d \sigma_{A A}^{h_{1}} / d^{2} p_{T}^{\text {trig }}},
$$

similarly to the direct-photon triggered FF $[12,13]$ in $\gamma$ jet events. Here, $z_{T}=p_{T} / p_{T}^{\text {trig }}$ and integration over $\left|y_{1,2}\right|<\Delta y$ is implied. In a simple parton model, the two jets should be exactly back-to-back. The initial parton transverse momentum distribution in our model will give rise to a Gaussian-like angular distribution. In addition, we also take into account transverse momentum smearing within a jet using a Gaussian distribution with a width of $\left\langle k_{\perp}\right\rangle=0.6$ $\mathrm{GeV} / c$. Hadrons from the soft component are assumed to be uncorrelated.

Shown in Fig. 4 are the calculated back-to-back correlations for charged hadrons in $A u+A u$ collisions as compared to the STAR data [25]. The same energy loss that is used to calculate single hadron suppression and azimuthal anisotropy can also describe well the observed away-side hadron suppression and its centrality dependence. In the data, a background $B\left(p_{T}\right)\left[1+2 v_{2}^{2}\left(p_{T}\right) \cos (2 \Delta \phi)\right]$ from uncorrelated hadrons and azimuthal anisotropy has been subtracted. The value of $v_{2}\left(p_{T}\right)$ is measured independently while $B\left(p_{T}\right)$ is determined by fitting the observed correlation in the region $0.75<|\phi|<2.24 \mathrm{rad}[25]$.

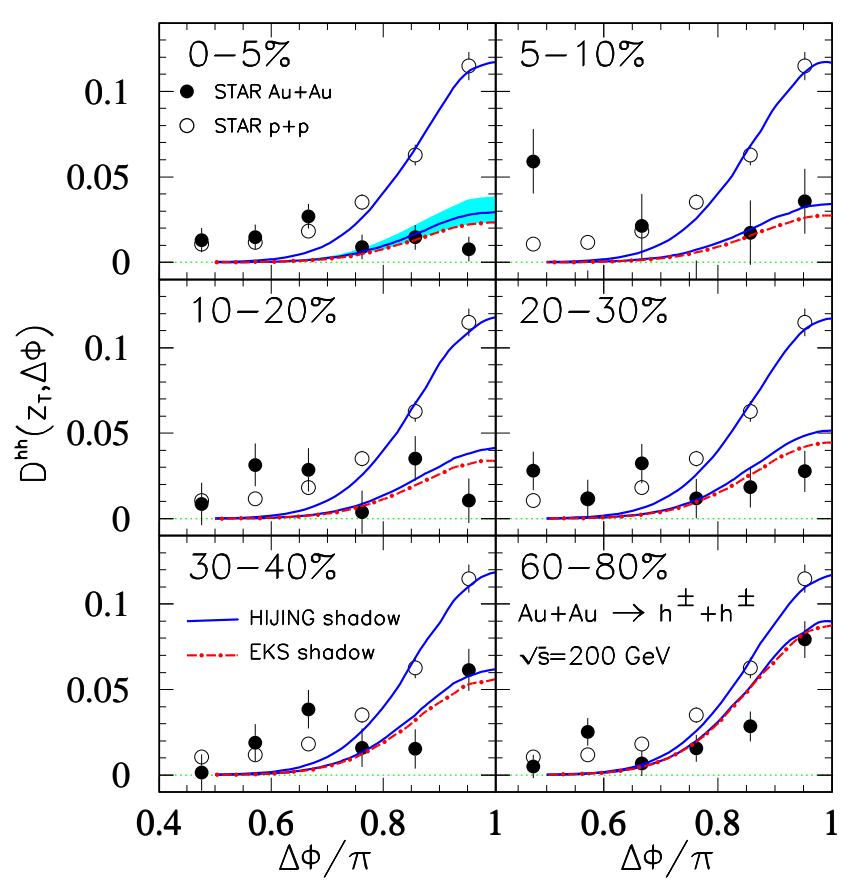

Figure 4. Back-to-back correlations for charged hadrons with $p_{T}^{\text {trig }}>p_{T}>2 \mathrm{GeV} / c, p_{T}^{\text {trig }}=4-6 \mathrm{GeV} / c$ and $|y|<0.7$ in $A u+A u$ (lower curves) and $p+p$ (upper curves) collisions as compared to the STAR[25] data.

With both the single spectra and dihadron spectra, the extracted average energy loss in this model calculation for a $10 \mathrm{GeV}$ quark in the expanding medium is $\langle d E / d L\rangle_{1 d} \approx$ $0.85 \pm 0.24 \mathrm{GeV} / \mathrm{fm}$, which is equivalent to $d E_{0} / d L \approx$ 
$13.8 \pm 3.9 \mathrm{GeV} / \mathrm{fm}$ in a static and uniform medium over a distance $R_{A}=6.5 \mathrm{fm}$. This value is about a factor of 2 larger than a previous estimate [22] because of the variation of gluon density along the propagation path and the more precise RHIC data considered .

\section{Summary}

In summary, with the recent measurements of high $p_{T}$ hadron spectra and jet correlations in $d+A u$ collisions at RHIC, the observed jet quenching in $A u+A u$ collisions has been established as a consequence of final state interaction between jets and the produced dense medium. The collective body of data: suppression of high $p_{T}$ spectra and back-to-back jet correlation, high $p_{T}$ anisotropy and centrality dependences of the observables, indicate that the cause of the jet quenching is due to parton energy loss rather than final state absorption of hadrons.

A simultaneous phenomenological study of the suppression of hadron spectra and back-to-back correlations, and high- $p_{T}$ azimuthal anisotropy in high-energy heavy-ion collisions within a single LO pQCD parton model incorporating current theoretical understanding of parton energy loss can describe the experimental data of $A u+A u$ collisions very well. With HIJING (EKS) parton shadowing, the extracted average energy loss for a $10 \mathrm{GeV}$ quark in the expanding medium is $\langle d E / d L\rangle_{1 d} \approx 0.85(0.99) \pm 0.24 \mathrm{GeV} / \mathrm{fm}$, which is equivalent to $d E_{0} / d L \approx 13.8(16.1) \pm 3.9 \mathrm{GeV} / \mathrm{fm}$ in a static and uniform medium over a distance $R_{A}=6.5$ $\mathrm{fm}$. This value is about a factor of 2 larger than a previous estimate [22] because of the variation of gluon density along the propagation path and the more precise RHIC data considered here .

\section{Acknowledgement}

This work is supported by the Director, Office of Energy Research, Office of High Energy and Nuclear Physics, Divisions of Nuclear Physics, of the U.S. Department of Energy under Contract No. DE-AC03-76SF00098

\section{References}

[1] Alan D. Martin, R. G. Roberts, W. J. Stirling, and R. S. Thorne, Eur. Phys. J. C23 73, 2002; J. Pumplin et al., JHEP 07012,2002 .

[2] T. Matsui and H. Satz, Phys. Lett. B 178, 416 (1986).
[3] X. N. Wang and M. Gyulassy, Phys. Rev. Lett. 68, 1480 (1992).

[4] X. N. Wang and X. F. Guo, Nucl. Phys. A 696, 788 (2001).

[5] X. F. Guo and X. N. Wang, Phys. Rev. Lett. 85, 3591 (2000).

[6] B. W. Zhang and X.-N. Wang, arXiv:hep-ph/0301195.

[7] M. Luo, J. Qiu, and G. Sterman, Phys. Lett. B279, 377 (1992); M. Luo, J. Qiu, and G. Sterman, Phys. Rev. D50, 1951 (1994); M. Luo, J. Qiu, and G. Sterman, Phys. Rev. D49, 4493 (1994).

[8] J. Osborne and X.-N. Wang, Nucl. Phys. A 710, 281 (2002) [arXiv:hep-ph/0204046].

[9] A. Airapetian et al. [HERMES Collaboration], Eur. Phys. J. C 20, 479 (2001); V. Muccifora [HERMES Collaboration], arXiv:hep-ex/0106088.

[10] R. Baier, Y. L. Dokshitzer, A. H. Mueller, S. Peigne, and D. Schiff, Nucl. Phys. B 484, 265 (1997)

[11] M. Gyulassy, I. Vitev, and X. N. Wang, Phys. Rev. Lett. 86, 2537 (2001).

[12] X. N. Wang, Z. Huang, and I. Sarcevic, Phys. Rev. Lett. 77, 231 (1996).

[13] X. N. Wang and Z. Huang, Phys. Rev. C 55, 3047 (1997).

[14] E. Wang and X. N. Wang, Phys. Rev. Lett. 87, 142301 (2001).

[15] X. N. Wang, Phys. Rev. C 61, 064910 (2000).

[16] X. N. Wang, arXiv:nucl-th/0305010.

[17] J. Binnewies, B. A. Kniehl, and G. Kramer, Z. Phys. C 65, 471 (1995).

[18] A. D. Martin, R. G. Roberts, W. J. Stirling, and R. S. Thorne, Eur. Phys. J. C 4, 463 (1998).

[19] S. Y. Li and X. N. Wang, Phys. Lett. B 527, 85 (2002).

[20] S. S. Adler et al. [PHENIX Collaboration], Phys. Rev. Lett. 91, 072303 (2003)

[21] J. Adams et al. [STAR Collaboration], Phys. Rev. Lett. 91, 072304 (2003)

[22] E. Wang and X. N. Wang, Phys. Rev. Lett. 89, 162301 (2002).

[23] K. Adcox et al., Phys. Rev. Lett. 88, 022301 (2002); S. S. Adler et al., Phys. Rev. Lett. 91, 072301(2003).

[24] C. Adler et al., Phys. Rev. Lett. 89, 202301 (2002); J. L. Klay, Nucl. Phys. A715, 733 (2003).

[25] C. Adler et al., Phys. Rev. Lett. 90, 082302 (2003) (I thank STAR collaboration for providing the data in $5-10 \%$ and $10-$ $20 \%$ centrality bins in addition to the published centrality bins). 